\title{
Linguagem e Cognição: A Referencialidade como um Construto Mental*
}

\author{
(Language and Cognition: Referentiality as a Mental Construct)
}

\author{
Lucia Maria Pinheiro Lobato \\ (Universidade de Brasília/CNPq)
}

\begin{abstract}
This paper is developed within the scope of the debate between internalist and externalist views of reference, and argues in favor of the internalist view, translated in terms of the relation Refer. By carrying out a contrastive analysis of data from Brazilian Portuguese and European Portuguese, it aims to demonstrate the existence of formal features which, under given circumstances, denote entities. It is proposed that the faculty of language deals with the concept of entity only as the result of formal features, and that formal features are responsible for the interpretation of an entity with respect to a given world.
\end{abstract}

KEY-WORDS: formal features; reference; entity; internalism E externalism.

RESUMO: Este artigo se insere no debate entre as visões internalista e externalista a respeito da referência, buscando argumentar empiricamente a favor da visão internalista, traduzida em termos da relação Referir. Por meio da análise dos dados extraídos do português do Brasil, em contraste com dados do português europeu, procura-se demonstrar que existem certos traços formais que, em dadas circunstâncias, denotam entidade. A proposta é que a faculdade de linguagem lida com o conceito de entidade, produzido por efeito de traços formais, e que esse conceito é interpretado como pertencendo a um dado mundo também pelo efeito de traços formais.

PALAVRAS-CHAVE: traços formais; referência; entidade; internalismo E externalismo.

* Este texto, póstumo e inédito, corresponde à conferência proferida por Lucia Lobato na $54^{\mathrm{a}}$ Reunião Anual da Sociedade Brasileira para o Progresso da Ciência (SBPC), em 2002. A seleção e edição do manuscrito deixado por Lucia Lobato foram feitas por Heloisa Salles, Rozana Naves, Eloisa Pilati e Helena G. Vicente.

D.E.L.T.A., 23:esp., 2007 (1-16) 


\section{Externalismo vs. Internalismo}

Não há dúvida de que as pessoas usam palavras para se referir a coisas e falar sobre elas. Há, no entanto, controvérsia a respeito de as palavras se referirem a essas coisas. A posição clássica a esse respeito diz que sim, os signos designam objetos do mundo. Essa visão externalista da referência se encontra em diferentes autores. Um deles é Frege. Na visão de Frege, um signo designa um objeto do mundo e tem um sentido que fixa a referência e é 'aprendido por todo mundo' que sabe a língua. Entender uma expressão é conhecer seu sentido numa 'língua pública compartilhada'. Além disso, cada pessoa tem uma imagem mental conectada com o sentido objetivo. Signo, sentido e referente são entidades externas, fora da mente/ cérebro. A imagem mental é o que é interno à mente/cérebro. Nessa visão, a idéia fundamental é que um signo escolhe um objeto no mundo ao qual se refere, e o faz de um modo determinado por seu sentido. De modo geral, pode-se dizer que essa posição vê a relação entre as palavras e as coisas como uma propriedade das palavras, intermediada pelo sentido.

Essa visão de que existe uma relação de referência entre expressões lingüísticas e objetos externos, intermediada pelo sentido, não é sem lógica. Consideremos a palavra 'gato' em português. Para se poder dizer que uma criança qualquer, em fase de aquisição do português, adquiriu essa palavra, é preciso que a criança tenha adquirido a habilidade de associá-la a um certo tipo de animal do mundo real da classe dos felinos. Assim, se uma tal criança folheia um livro, aponta para a imagem de um gato e pronuncia a palavra 'cachorro', temos certeza de que ainda não adquiriu a palavra 'gato'. Ela não sabe o que a palavra 'gato' significa. Uma criança que sabe o que 'gato' significa necessariamente associa a palavra 'gato' à imagem de um certo tipo de felino e nunca à imagem de um canino. Em resumo, palavras como 'gato' são usadas para referência a coisas e para se falar sobre essas coisas, e adquirir tais palavras implica necessariamente aprender essa relação da palavra com a coisa. Como a coisa, nesse caso, é algo externo à mente/cérebro, tem de haver, segundo esse raciocínio, uma relação de referência ligando as palavras concretas às coisas do mundo externo que elas denotam.

Chomsky $(1993,2000)$ se posiciona contra qualquer visão externalista da referência e defende uma visão internalista (ou individualista). ${ }^{1}$ Segundo

\footnotetext{
1 Para um minucioso exame crítico dos diferentes argumentos a favor do externalismo e contra o internalismo e para uma argumentação a favor do internalismo, V. Chomsky (2000), assim como as obras ali citadas.
} 
essa posição, as palavras não se referem às coisas; antes, são as pessoas que usam as palavras para se referir às coisas. Portanto, a referência não é uma propriedade das palavras, e sim uma propriedade que os falantes atribuem às palavras. Nessa perspectiva, referir é estritamente um processo computacional mental, ligado a um dos sistemas de desempenho da faculdade de linguagem. $\mathrm{Na}$ visão de Chomsky, a faculdade de linguagem, em qualquer estágio de seu desenvolvimento, inclui um sistema cognitivo e sistemas de desempenho. O sistema cognitivo armazena informações que são acessadas pelos sistemas de desempenho e usadas para articular, interpretar, expressar o pensamento, fazer perguntas, referir, e assim por diante. Ambos os tipos de sistemas estão dentro da mente/cérebro. Portanto, referir é um processo internalista.

A proposta internalista é que existe uma relação Referir (relação-R) "entre expressões lingüísticas e algo mais, entidades extraídas de algum domínio estipulado D (talvez valores semânticos)” (Chomsky 2000: 3839). Em que consiste essa relação-R? Chomsky não dá detalhes a respeito. Somente observa (p. 39; p. 202 N. 6) que deve ser tratada pela sintaxe.

A hipótese internalista da referência tem suscitado críticas. Jerry Fodor (2000), por exemplo, diz:

Apesar de ser verdade que muito da fonologia e da sintaxe gerativas é Internalista, grande parte da semântica e da psicologia cognitiva não é. [...] Na verdade, duvido que o Internalismo, como Chomsky o concebe, seja verdadeiro.

Para Fodor, pode-se falar em Internalismo em semântica quando se trata de raciocínio lógico, mas não quando se trata de percepção e memória:

Ver uma cadeira é plausivelmente uma relação entre a mente, de um lado, e a cadeira, de outro. Sem dúvida, muito do que acontece quando alguém vê uma cadeira acontece, literalmente, na cabeça da pessoa, mas a cadeira não está na mente (nem no cérebro tampouco, pressupondo que é diferente); se elas estivessem dentro da mente, cadeiras não serviriam para sentar. A memória funciona do mesmo modo. Grande parte do que acontece quando alguém se lembra, acontece no hipocampo, de acordo com o boato corrente. Mas é da "vovó" que a pessoa se lembra, e a vovó não é mental.

Isso importa, porque a essência do quadro de Chomsky/Turing é que os processos mentais são computações que o cérebro executa, e que o cérebro está dentro não se questiona. Se percepção, memória e coisas desse tipo são parte daquilo que se supõe que a psicologia deve explicar, a conclusão parece ser que a computação não pode ser toda a história sobre a mente. 
Chomsky acha que uma teoria computacional do cérebro é toda a ciência da mente ou da língua que possa existir. Externalistas acham que é somente parte de uma história muito mais longa sobre as interações mente/mundo do tipo que a percepção e o relembrar aparentemente exemplificam.

O objetivo deste artigo é argumentar empiricamente a favor da visão da referência, traduzida em termos da relação Referir. A proposta é que a faculdade de linguagem lida com traços abstratos que traduzem, entre outros, o conceito de entidade. O conceito de entidade é a propriedade referencial essencial dos nomes. Esse conceito está presente nos verbetes dos nomes, que o usam na relação-R. O mesmo conceito se encontra nos verbetes dos adjetivos, mas não como uma propriedade referencial e sim como uma propriedade combinatória: o conceito de entidade presente nos verbetes dos adjetivos permite que esses itens se combinem com nomes. $\mathrm{O}$ conceito de entidade é também formado na sintaxe. É o caso das formas pronominais, como, por exemplo, as manifestas na posição de sujeito. Considero que, em qualquer caso, o conceito de entidade é construído com o uso de traços formais. Não vou tratar aqui da questão de quais são os traços formais. Vou simplesmente procurar demonstrar, com dados empíricos extraídos do português do Brasil, em contraste com o português europeu, que esses traços existem e que, em dadas circunstâncias, denotam entidade. Além de a mente construir o conceito de entidade, também constrói a sua interpretação como entidade de um certo mundo material ou psicológico.

Em resumo, a proposta é que a faculdade de linguagem lida com o conceito de entidade, produzido por efeito de traços formais, e que esse conceito é interpretado como pertencendo a um dado mundo também pelo efeito de traços formais. Essa interpretação é que estabelece a relação entre as expressões lingüísticas e entidades extraídas de algum domínio estipulado D, segundo a proposta que estou fazendo. Por que 'domínio D' e não mundo externo? Porque, de fato, o conceito de entidade do mundo material, por exemplo, é efeito de uma certa interpretação do conceito de entidade. A mente cria estruturas que nos levam a interpretar as entidades associadas aos nomes como existentes em um certo mundo (mundo psicológico ou mundo material, mundo real e contemporâneo ao ato de fala ou mundo possível, e assim por diante). Referir é, então, um processo estritamente internalista. 
A argumentação vai usar dados do português do Brasil (PB), em contraste com dados do português europeu (PE). A próxima seção é dedicada à apresentação de evidências do $\mathrm{PE}$ e do $\mathrm{PB}$ de que a sintaxe não somente lida com o conceito de entidade, mas também o introduz.

\section{Perguntas Qu- e Sentenças com Sujeito Topicalizado no PE e no PB}

Eugenia Duarte (1995) mostrou que as perguntas Qu- com sintagma Qu- deslocado e as sentenças com topicalização de sujeito são contexto de variação entre o $\mathrm{PE}$ e o $\mathrm{PB}$ com relação à manifestação de sujeito pronominal pré-verbal: o PB tende a manifestar sujeito pronominal pré-verbal nessas estruturas, contrariamente ao $\mathrm{PE} .^{2}$

\subsection{O PB Tende a Preencher a Posição de Sujeito Dessas Sentenças}

Os exemplos em (1)-(2) ilustram o fato de o PB preencher a posição de sujeito de sentenças interrogativas com sintagma Qu- deslocado e de sentenças com topicalização de sujeito:

(1) a. Que disco ${ }_{1}$ (que) ele $\mathrm{O}_{\text {que }_{1}}$ (que) ele ${ }_{2}$

Que carro $_{1}$ (que) o Henrique 2 ganhou? $\mathrm{O}_{\text {que }}$ (que) o Henrique ${ }_{2}$ ganhou?

b. *Que disco

ganhou?

$* \mathrm{O}$ que ganhou?

(2) a. Esta mesa ${ }_{1}$, ela 2 está suja.

b. *Esta mesa, está suja.

\footnotetext{
2 Seguindo uma certa tradição de análise, E. Duarte (1995) distingue topicalização de deslocamento à esquerda (DE). Topicalização diz respeito a deslocamento de objeto, e DE, a deslocamento de sujeito. Portanto, o que estou chamando de 'topicalização de sujeito' é rotulado de DE por Duarte. Por exemplo, Chomsky (1977) rotula de deslocamento à esquerda casos como 'As far as John is concerned, I will never believe the claims that have been made about him'. Para ele, a distinção entre DE e topicalização diz respeito a haver, ou não, movimento envolvido - na topicalização há, mas no DE não, e o critério para saber se há ou não há movimento é o de haver, ou não, satisfação às restrições de ilha.
} 
No entanto, nem sempre o PB licencia o sujeito manifesto em interrogativas com sintagma Qu- deslocado, como bem o ilustra a agramaticalidade de (3b). Em (3a), o sintagma Qu- deslocado é o sujeito. Em (1), é o objeto. Concluo que a exigência para a manifestação de sujeito em oração com sintagma Qu- deslocado é que esse sintagma não seja sujeito.

(3) a. Quem 1 (que) - viu ele $e_{2}$ sair?

b. $*$ Quem $_{1}$ (que) ele ${ }_{1}$ viu Pedro 2 sair?

$\mathrm{O}$ vestígio de sintagma $\mathrm{Qu}$ - deslocado é interpretado como uma variável. Logo, não pode haver a manifestação de sujeito pronominal quando a posição original de sujeito contém uma variável (var) ligada ao sintagma $\mathrm{Qu}$ - deslocado.

(4) a. Quem ${ }_{1}$ (que) $\operatorname{var}_{1}$ viu ele $_{2}$ sair?

b. *Quem 1 (que) ele viu Pedro sair? $_{1}$

Como mostra o par em (5), caso não haja esse tipo de ligação, a lexicalização é forçada:

(5) a. Quem 1 (que) $\underline{\text { ele }}_{2}$ viu var $_{1}$ sair?

b. $*$ Quem $_{1}$ (que) $\varnothing$ viu $\operatorname{var}_{1}$ sair?

Finalmente, parece tratar-se de restrição independente de contigüidade, dada a agramaticalidade de (6a):

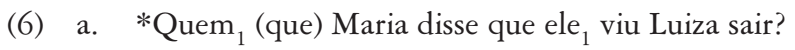

b. Quem ${ }_{1}$ (que) Maria disse que $v^{1}{ }_{1}$ viu Luiza sair?

Considero que a sintaxe oracional lida com três núcleos funcionais (C, T, Asp), sendo (7) a estrutura sintática básica:

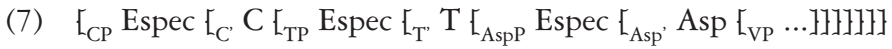

A análise convencional para deslocamento Qu- posiciona o sintagma $\mathrm{Qu}$ - deslocado em Espec,CP, como na estrutura simplificada em (8):

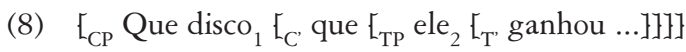

Para sentenças com topicalização, há mais de uma tendência de análise. Uma delas trata tópicos como adjuntos de uma projeção funcional sentencial IP (cf. I. Duarte, 1987; E. Duarte, 1995: 71). A outra tendência de análise considera que tópicos projetam TopP (cf. Chomsky, 1977; Benincà, 
1995; Raposo \& Uriagereka, 1996; entre outros). Estou propondo um terceiro tipo de análise: tópicos não projetam TopP e nem projetam estrutura de adjunção; antes, projetam CP; isto é, tópicos ocorrem em Espec,CP, do mesmo modo que sintagmas Qu-. Uma evidência a favor de tópicos ocorrerem na mesma posição de sintagmas Qu- deslocados é exatamente o efeito que essas estruturas têm sobre Espec,TP no PB. Isto é, a hipótese de haver uma estrutura em comum tem poder explicativo em relação aos fatos do PB: em todos esses contextos, é o núcleo funcional $\mathrm{C}$ o causador da manifestação fonológica em Espec,TP.

Aceitando essa análise sintagmática, o que os dados do PB parecem nos mostrar é que $C$ tem efeito licenciador de lexicalização em Espec,TP (sob condições específicas da gramática em questão). A comparação de (1a) com (2a), repetidos abaixo, leva a concluir que, na situação descrita, a lexicalização em Espec,TP é provocada por C, quer C tenha sua informação inerente manifestada sob forma da integrante 'que' (1a), ou não (2a). Portanto, C afeta Espec,TP no PB mesmo que seu traço inerente receba manifestação fonológica:

(1') a. [Que disco ${ }_{1} \mathrm{C}$-que [ele ${ }_{2}$ ganhou $\left.]\right\}$ ?

(2') a. [Esta mesa ${ }_{1}$ C- $\varnothing \quad\left[\right.$ ela ${ }_{1}$ está suja $]$ ].

Em resumo, segundo a perspectiva teórica que estou tomando, C tem o efeito de provocar manifestação fonológica em Espec,TP quando Espec, CP está preenchido, sob condições precisas. No $\mathrm{PB}$, a condição é não haver ligação do tipo "sintagma Qu- - Variável” entre as duas posições.

\subsection{O PE Não Tende a Preencher a Posição de Sujeito Dessas Sentenças}

Os fatos do PE a respeito de licenciamento de manifestação de sujeito pronominal pré-verbal em estruturas interrogativas Qu- e estruturas com topicalização de sujeito são bem diferentes dos do PB. Essa manifestação não é sistematicamente agramatical no $\mathrm{PE}$, mas é submetida a severas restrições.

Quanto a interrogativas Qu-, mesmo no caso da presença de sintagma lexical não-pronominal pleno, há restrições ao uso de sujeito manifesto na contigüidade à direita do sintagma Qu-, como mostram os exemplos (9)(10) (cf. Âmbar, 1992): 
(9) a. *O que o Henrique comprou?

b. O que é que o Henrique comprou? (PE coloquial)

c. O que comprou o Henrique?

(PE literário)

d. O que O HENRIQUE comprou?

e. O QUE o Henrique comprou?

(10) a. ?Que disco o Henrique comprou?

b. Que disco é que o Henrique comprou? (PE coloquial)

c. Que disco comprou o Henrique?

(PE literário)

d. Que disco O HENRIQUE comprou?

e. QUE DISCO o Henrique comprou?

(9a) e (10a) mostram que essa contigüidade resulta em agramaticalidade no caso de o sintagma Qu- não conter palavra lexical interna, e marginalidade no caso de conter. (9d,e) e (10d,e) mostram que essa ordem só se torna gramatical se um dos dois constituintes - o sintagma Qu- deslocado ou o sujeito lexical - recebe acento contrastivo. (9c) e (10c) evidenciam que o uso de $\mathrm{V}$ em $\mathrm{C}$ é a estratégia literária do $\mathrm{PE}$ para estruturas interrogativas Qu-. (9b) e (10b), finalmente, mostram que a estratégia coloquial do PE para essas estruturas é o uso de 'ser que'.

No caso de sujeito pronominal, E. Duarte (1995) aponta que seu uso é agramatical em interrogativas Qu- com sintagma Qu-deslocado, como mostra (11a), havendo preferência pelo uso de sujeito nulo:

(11) a. *Que disco ele comprou?

b. Que disco comprou?

Esse exemplo contrasta com (10a). E. Duarte (1995) registrou, no entanto, o exemplo em (12), e observa que se trata de pronome sujeito de primeira pessoa:

(12) Como eu hei de lhe dizer?

Se de fato (11a) é agramatical no PE, concluo que o deva ser numa leitura não-contrastiva, e que (12) é gramatical numa leitura enfática ou contrastiva para o sujeito. Isto é, (12) estaria em paralelo com (9d,e):

(9) d. O que O HENRIQUE comprou?

e. O QUE o Henrique comprou?

(12') a. Como EU hei de lhe dizer?

b. COMO eu hei de lhe dizer? 
Nesse caso, (11a) deve ser gramatical numa leitura contrastiva:

(11') a'. Que disco ELE comprou?

a". QUE DISCO ele comprou?

Em resumo, sendo os dados assim, o PE não licencia sujeito pronominal manifesto na adjacência direita de um sintagma Qu- deslocado, mesmo sem uma relação Operador-Variável entre eles, a não ser que o sujeito pronominal tenha leitura enfática ou contrastiva.

Quanto às estruturas com topicalização de sujeito, a diferença entre o $\mathrm{PE}$ e o $\mathrm{PB}$ é que o PB manifesta livremente um pronome resumptivo na posição original do sujeito deslocado, à esquerda do verbo, quer em orações matrizes, quer em encaixadas, e sem exigir referência prévia no discurso ao argumento sujeito, de modo que tanto (13) quanto (14a,b,c) são igualmente gramaticais em PB. O PE, no entanto, só aceita sujeito topicalizado com resumptivo manifesto sob condições bem precisas: (a) em oração matriz, mas não em encaixada, de modo que (13) é agramatical; e (b) em oração matriz com o contexto contrastivo, daí que (14a) é agramatical, e (14b) só é gramatical num contexto contrastivo, como (14c). ${ }^{3}$

(13) Eu acho que o povo brasileiro, ele tem uma grave doença. (PE * PB OK)

(14) a. O Edmilson, ele está? (PE *, PB OK) b. O Pedro, ele telefonou. (PE */OK, dependendo do contexto; PB OK) c. O Pedro, ele telefonou, a Maria não sei.

(PE OK, PB OK)

Outros exemplos em que o resumptivo ligado ao sujeito é gramatical são os em (15) e (16):

(15) A: Quem comeu o quê?

B: A Maria, ela comeu a sopa.

(PE OK)

(16) A: Quem foi à praia?

B: O Pedro, ele foi, os outros não sei.

(PE OK)

Por outro lado, se o resumptivo é pós-verbal, a sentença é gramatical no $\mathrm{PE}$, mas não no $\mathrm{PB}$, fato devido ao uso da estratégia de $\mathrm{V}$ para $\mathrm{C}$ no $\mathrm{PE}$, mas não no $\mathrm{PB}$ :

(17) Pedro, leu ele o livro.

$(\mathrm{PE} \mathrm{OK}, \mathrm{PB} *)$

3 Sobre o conjunto de dados de (13) a (17), vejam-se Costa (2000) e Costa \& Galves (2001). 
As análises que têm sido propostas para os contrastes em (13)-(14) entre o PE e o PB consideram que as diferenças se explicam em termos da posição estrutural do sujeito nas duas gramáticas (cf. Costa \& Galves, 2001). A análise que proponho fornece uma explicação diferente, baseada em outro pressuposto: o de que os núcleos funcionais sintáticos exercem diferentes papéis na sintaxe oracional nas gramáticas do $\mathrm{PE}$ e do $\mathrm{PB}$.

\subsection{A Sintaxe Cria o Conceito de Entidade}

No $\mathrm{PB}$, existem diferentes evidências que favorecem a hipótese de $\mathrm{C}$ ser licenciador de lexicalização na posição de sujeito. Aceitando essa hipótese, a análise que faço é que $\mathrm{C}$ licencia livremente Espec,TP no PB, mesmo estando Espec, $\mathrm{CP}$ ocupado por material lexical, desde que não haja Variável em Espec,TP.

$\mathrm{O} \mathrm{PE}$, ao contrário, em situações não-contrastivas, não usa $\mathrm{C}$ para licenciar o sujeito, tanto assim que é uma língua de sujeito nulo. Há diferentes alternativas de análise para os casos em que há licenciamento da manifestação fonológica de resumptivo em Espec,TP ligado ao DP em Espec, $\mathrm{CP}$, no PE. A primeira é pressupor que DPs tópicos são licenciados por $\mathrm{C}$ no $\mathrm{PE}$, em estruturas não-contrastivas, e considerar que nos exemplos gramaticais esse DP está independentemente licenciado, e não precisa usar $C$ para ser licenciado. Segundo essa alternativa de análise, (13’) é agramatical no $\mathrm{PE}$ porque $\mathrm{C}$ licencia 'o povo brasileiro' em Espec, $\mathrm{CP}$, e não há licenciador para o pronome 'ele' em Espec,TP:

$\left(13^{\prime}\right) * E_{C P} E_{T P}$ Eu acho $E_{C P}$ que-C $E_{C P}$ o povo brasileiro $C, E_{T P}$ ele tem $E_{A s p P}$ uma grave doença []] ]].

Em (14'a), igualmente, C licencia a ocorrência do material lexical em Espec,CP, e não há núcleo funcional licenciando a manifestação de 'ele' em Espec,TP.

(14') a. $*\left[_{\mathrm{CP}} \mathrm{O}\right.$ Edmilson $\mathrm{C}, \mathrm{L}_{\mathrm{TP}}$ ele está $]$ ?

Nos exemplos (15B) e (16B), em que o resumptivo ligado ao sujeito é gramatical, fica claro o que licencia essa ocorrência.

(15) A: Quem comeu o quê?

B: A Maria, ela comeu a sopa. 
(16) A: Quem foi à praia?

B: O Pedro, ele foi, os outros não sei.

Nesses dois exemplos, a pergunta já informa sobre a existência de entidade do mundo real, argumento externo do verbo, e indaga sobre a identidade dessa entidade (no caso de (15), indaga, mais precisamente, sobre a relação 'quem / o quê'). A gramaticalidade de (15) e (16) se deve, então, ao fato de $\mathrm{C}$ não precisar licenciar Espec, $\mathrm{CP}$, dada a informação sobre existência de entidade argumento externo do verbo na pergunta, podendo, então, licenciar a interpretação de existência de entidade em Espec,TP. Há um proble$\mathrm{ma}$, no entanto. Por que considerar que a estrutura argumental da pergunta está licenciando o tópico e não o sujeito? Afinal, na estrutura da pergunta, temos um sujeito, e não um tópico. Um outro problema com essa análise é que há evidências empíricas de que, em certos contextos não-contrastivos (precisamente em orações relativas, quer dizer, num contexto que não é de topicalização), o PE usa C para licenciar TP.

Uma outra alternativa, que vou aceitar, é considerar que o fornecimento, na pergunta, da estrutura argumental da sentença resposta, força a derivação se iniciar por $\mathrm{CP}$, o que, por sua vez, faz $\mathrm{C}$ ter efeito sobre Espec,TP. Nessa alternativa, o licenciamento de Tópico é somente estrutural, sem descarga do traço de $\mathrm{C}$ sobre esse DP. Na primeira alternativa, o traço de $\mathrm{C}$ era usado sobre Espec,CP, daí não poder ser usado sobre Espec,TP. Esta segunda alternativa é, então, compatível com o fato de, em certos contextos não-contrastivos, como é o caso das relativas, o $\mathrm{PE}$ usar $\mathrm{C}$ para licenciar TP.

Há evidência independente a favor dessa hipótese. Em estruturas relativas, como apontado por E. Duarte (1995), a manifestação de sujeito pronominal é não somente permitida, mas até favorecida, no PE. A condição para essa manifestação é que não haja correferência entre o núcleo e a posição de sujeito, da relativa (numa interpretação estendida da análise de E. Duarte), como nos exemplos em (17), retirados de E. Duarte (1995: 14): ${ }^{4}$

\footnotetext{
4 E. Duarte apontou (pp. 12-14, 60, 132-133) que as relativas são contexto estrutural favorecedor, e o único contexto estrutural favorecedor, de manifestação de sujeito pronominal no PE. Segundo ela, esse efeito depende de não haver relação de correferência entre o sujeito da relativa e o sujeito da matriz. Portanto, ao dizer que o fator relevante é a não-correferência entre o sujeito da relativa e o NP/DP núcleo da relativa, estou fazendo uma análise diferente dos dados. As duas análises não se excluem.
} 
(17) a. Aquilo que eu desejo é nunca ficar doente.

b. Aquelas coisas que nós ouvimos.

Há uma generalização que surge da comparação desse fato com os fatos do PB apontados, e quero captá-la. Para isso, é preciso considerar que o pronome relativo ocorre em C. Já foi levantada a hipótese de o relativo ser um complementador no $\mathrm{PB}$, mas nem sempre um complementador. Segundo Tarallo (1983) e Negrão (1999), o relativo ocupa a posição C nas relativas com resumptivo sujeito correferente com o núcleo da relativa ('uma mulher que ela é linda'), e a de Espec,CP nas relativas sem resumptivo ('uma mulher que é linda'). Estou analisando o relativo como ocorrendo em $\mathrm{C}$, em relativas com ou sem resumptivo. A diferença entre um relativo e um complementador tem a ver com os seus traços inerentes, e não com a sua posição estrutural: um complementador representa somente um núcleo funcional, $\mathrm{C}$, mas um relativo pode representar mais do que o núcleo $\mathrm{C}$, já que pode incorporar informação lexical (quem $=$ alguém; onde $=$ lugar, $\mathrm{e}$ assim por diante). $\mathrm{O}$ ônus desta proposta é que tenho de chegar a uma nova análise para as estruturas relativas sem resumptivo. Segundo a abordagem clássica, a presença da variável em relativas é explicada em termos de ligação Operador-Variável, estando o Operador em Espec,CP e a Variável na posição original do vestígio. É possível uma explicação para a diferença entre presença de Variável, para as relativas sem resumptivo, e presença de pronominal, para as relativas com resumptivo, que não faça apelo a uma relação de ligação entre Operador em Espec,CP e Variável em Espec,TP. No entanto, a análise dessa questão está fora do escopo deste trabalho. Vou simplesmente aceitar que o relativo ocupa sempre a posição C. Kato (1993) propõe uma abordagem diferente da de Tarallo (1983) e Negrão (1999): o relativo sempre está em Espec,CP e há um traço +WH em C. A análise que estou propondo diverge portanto também da abordagem de Kato (1993).

Essa perspectiva de análise me permite concluir que o PE também licencia manifestação de sujeito em Espec,TP por efeito de C, em contexto em que o Espec,CP imediatamente mais alto está preenchido. A diferença entre o PE e o PB está nas condições sob as quais $C$ tem efeito de licenciamento fonológico em Espec,TP: para o PE, o único contexto não-contrastivo de licenciamento favorecido é o de relativas sem correferência entre Espec,CP e Espec,TP. Dados os contrastes e as semelhanças com o PB, parece claro qual é o fator que está influenciando a manifestação de sujeito 
em Espec,TP no PE: são as próprias características derivacionais das relativas. Nas relativas, CP é um constituinte interno à expressão nominal, já que uma relativa é um adjunto de um NP (relativa restritiva) ou de um DP (relativa apositiva). O constituinte, como um todo, é uma expressão nominal, e não uma sentença. Logo, na derivação de uma relativa, é razoável imaginar que o chamado núcleo da relativa é derivado antes da relativa, por ser a relativa um adjunto desse núcleo. Sendo assim, ao se chegar na derivação da relativa, ela vai se dar de cima para baixo. Isto é, vai operar iniciando por CP. É essa a condição para C operar sobre Espec,TP, segundo a análise que estou fazendo. Em resumo, as relativas são um contexto favorecedor de manifestação de sujeito pré-verbal no PE porque se trata de um contexto em que a derivação da sentença tem início em CP.

Vimos que o resumptivo é gramatical no $\mathrm{PE}$, mas não no $\mathrm{PB}$, se pósverbal:

(18) Pedro, leu ele o livro.

$(\mathrm{PE} \mathrm{OK}, \mathrm{PB} *)$

(18') $\mathrm{L}_{\mathrm{CP}}$ Pedro C, $\mathrm{L}_{\mathrm{TP}} \mathrm{t}$ leu-T $\mathrm{L}_{\mathrm{AspP}}$ ele Asp- $\mathrm{t} \mathrm{L}_{\mathrm{VP}}$ o livro $\left.\left.]\right\}\right]$.

Esse fato me faz concluir que no PE, nesse tipo de estrutura, V-T licencia o sujeito em Espec,AspP, e Asp-t licencia o objeto em Espec,VP. C não licencia Espec,TP em virtude de, no $\mathrm{PE}$, a derivação, em estruturas não-contrastivas, se iniciar a partir de Asp, e não de C.

Em resumo, segundo a análise que estou fazendo, a gramática do PE, quando não forçada a fazer diferentemente, não inicia as derivações por $\mathrm{C}$ (antes o faz por Asp), e somente inicia por $\mathrm{C}$ quando forçada a assim o fazer, o que ocorre em estruturas relativas, devido à posição de adjunto da oração, e em estruturas não-relativas contrastivas, devido ao fato de o contexto discursivo anterior já fornecer a estrutura argumental da sentença, o que torna a derivação iniciada por AspP redundante, considerando que nesse caso a estrutura argumental em VP teria de estar sendo levada em conta. Está aí a explicação para o uso que o PE faz do pronome resumptivo em estruturas com sujeito topicalizado: esse tipo de resumptivo ocorre por licenciamento de C sobre Espec,TP; e numa derivação iniciada por Asp, Asp licencia o sujeito em VP; os contextos contrastivos licenciam a ocorrência do resumptivo porque, nesse caso, a derivação começa por $\mathrm{C}$, e não por Asp, uma vez que a estrutura argumental já é dada no contexto discursivo anterior. $\mathrm{O}$ uso regular que o $\mathrm{PB}$ faz do resumptivo, em estruturas 
com sujeito topicalizado, se explica em função de o PB iniciar sempre a derivação sintática por C.

Por que o PE inicia as derivações por Asp e o PB por C? Porque o PE faz as derivações sintáticas lendo os traços temáticos dos itens lexicais da numeração, daí ter de partir de VP, fazer uma derivação de baixo para cima, e só poder fazer a derivação de cima para baixo nos casos estritos em que o contexto discursivo anterior fornece a estrutura argumental. O PB faz as derivações sistematicamente de cima para baixo porque toma a estrutura conceptual como pano-de-fundo para a derivação sintática.

\section{Construção do Conceito de Entidade e da Interpretação de Existência no Mundo}

Voltando ao conceito de entidade, como e por que os dados do PE e do PB sobre interrogativas Qu- e sobre topicalização de sujeito são evidência a favor da tese internalista de referência? Esses dados favorecem a hipótese de construção mental do conceito de entidade pela sintaxe porque se trata de duas gramáticas diferentes, mas muito próximas, e que variam no licenciamento da projeção sintática do sujeito pronominal.

No caso do PB, interpreto o fato de C, nas circunstâncias que vimos, forçar a projeção de um sujeito em Espec,TP como advindo do efeito do traço abstrato de $\mathrm{C}$ sobre Espec,TP, juntamente com o fato de a posição afetada pelo traço ser exatamente Espec,TP, que está, então, sob duplo efeito - o efeito estrutural de T e o efeito de descarga do traço de C. Não estou querendo dizer que $\mathrm{C}$ cria a interpretação de entidade. Estou querendo dizer que o nível (ou plano) em que a gramática do PB leva a derivação para a manifestação fonológica é um nível tal que torna o conceito de entidade acessível ao traço de $\mathrm{C}$. Afinal, de onde é que são tiradas todas as ocorrências de sujeito pronominal no PB e que são agramaticais no PE? Como explicar a retomada pronominal do tópico sujeito, na posição de sujeito, que fazemos com tanta consistência e sem restrições? A cada vez que fazemos essa retomada, estamos atualizando o conceito de entidade.

No caso do PE, a construção do conceito de entidade na sintaxe oracional se faz de modo diferente, como os dados mostram tão claramente, pois não se faz inicialmente por C. Mas, de qualquer maneira, há um li- 
cenciamento sintático, de um modo também bem particular - é feito por C quando isso é forçado pela informação lexical (construção enfática do sujeito pronominal em interrogativas Qu-) ou pela informação discursiva prévia (caso da retomada pronominal do sujeito topicalizado, quando o contexto discursivo anterior licenciou a estrutura argumental verbal).

Em resumo, o PB cria o conceito de entidade na posição de sujeito de modo gramaticalizado, por meio do uso C. Daí a sistematicidade da ocorrência de sujeitos manifestos no PB. No PE, C só age sobre Espec,TP quando a informação lexical abre essa via, não sendo de uso automático como no PB.

\section{REFERÊNCIAS BibLIOGRÁficAS}

Ambar, Manuela. 1992. Para uma Sintaxe da Inversão Sujeito-Verbo em Português. Lisboa: Colibri.

Benincà, Paola. 1995. Complement clitics in Medieval Romance. The Tobler-Mussaffa Law. In: A. BATTYE and I. RoBerTs (eds.) Clause Structure and Language Change. Oxford: Oxford University Press, pp. 325-344.

Costa, João. 2000. Multiple focus, apparent optionality and subject position. Comunicação apresentada durante o II Congresso Internacional da Abralin. Fortaleza.

Costa, J. \& C. Galves. 2001. Sujeitos periféricos em duas variedades do português. Comunicação apresentada durante o II Congresso Internacional da Abralin. Fortaleza.

Chomsky, N. 1977. On wh-movement. In: P. Culicover, T. Wasow and A. Akmajian (eds.) Formal syntax. New York: Academic Press. 71-132. . 1993. Language and thought. Wakefield, Rhode Island, Moyer Bell. .2000. New horizons in the study of language and mind. Cambridge: Cambridge University Press. 2005. On phases. Ms. MIT.

Duarte, M. E. L. 1995. A perda do Princípio 'Evite Pronome' no Português Brasileiro. Tese de Doutorado. Campinas, Unicamp.

Dunkte, I. 1987. A construção de topicalização na Gramática do Português: Regência, ligaçãa e condições sobre movimento. Dissertação de Mestrado. Universidade de Lisboa. 
FODOR, J. 2000. It's all in the mind. TLS 23.06.2000.

Kato, M. A. 1993. Recontando a história das relativas em uma perspectiva paramétrica. In: I. RoBerTs and M. KaTo (orgs.) Português Brasileiro: Uma viagem diacrônica. Campinas: Editora da Unicamp. 223-261.

Negrão, E. 1999. O Português Brasileiro: Uma língua voltada para o discurso. Tese de Livre-Docência, inédita. São Paulo, USP.

Raposo, Eduardo \& Juan UriagereKa. 1996. Indefinite 'se'. Natural Language and Linguistic Theory 14: 749-810.

Tarallo, Fernando. 1983. Relativization Strategies in Brazilian Portuguese. Tese de Doutorado, inédita. University of Pennsylvania, EUA. 